\title{
Niveles de participación de niños, niñas y adolescentes en investigaciones de educación ambiental en Hispanoamérica (1999-2019)
}

\author{
Levels of Participation of Children and Adolescents \\ in Research of Environmental Education in Hispanic \\ America (1999-2019)
}

\author{
Gabriel Prosser Bravo ${ }^{1}$, Iván Romo-Medina ${ }^{2}$ y \\ Rodrigo Rojas-Andrade ${ }^{3}$ \\ 1,3 Universidad Academia de Humanismo Cristiano \\ ${ }^{2}$ Universidad de Chile
}

\begin{abstract}
Resumen
Históricamente se ha reconocido en la educación ambiental una tradición participativa y horizontal. Pese a ello, las investigaciones empíricas describen bajos niveles de inclusión de niños, niñas y adolescentes en este tipo de educación y en su rama investigativa: son escasas las investigaciones que miden esta intervención, limitándose a describir casos de estudio. Para precisar aquello, se realizó una revisión bibliográfica sistematizada de las investigaciones de educación ambiental escritas en espańol desde 1999 a 2019, en las cuales participaron niños, niñas y adolescentes en diferentes grados de involucramiento. Se codificó el nivel de participación por medio de la escalera de Roger Hart, la cual describe ocho peldaños distintos. Esto fue correlacionado mediante análisis estadísticos y pruebas no paramétricas con el año y país de publicación, el tipo de educación y la metodología empleada. Los resultados indican un aumento progresivo de los niveles de participación infantil y adolescente de 1999 a la fecha, como también niveles más altos en la educación ambiental no formal, si se consideran metodologías cualitativas para investigar (autores de Argentina y Cuba). Se discute la importancia de repensar el lugar de niños, nińas y adolescentes en el desarrollo de la educación e investigación ambiental.
\end{abstract}

Palabras clave: educación ambiental, investigación en educación ambiental, participación adolescente, participación infantil, revisión bibliográfica sistematizada.

Correspondencia a:

Gabriel Prosser Bravo

Escuela de Psicología de la Universidad Academia de Humanismo Cristiano,

Avenida Condell 343, Providencia, Santiago de Chile.

gabrielprosserb@gmail.com.

(C) 2020 PEL, http://www.pensamientoeducativo.org - http://www.pel.cl

ISSN:0719-0409 DDI:203.262, Santiago, Chile doi: 10.7764/PEL.57.2.2020.8 


\title{
Abstract
}

\begin{abstract}
Historically, a participatory and horizontal tradition has been recognized in environmental education. In spite of this, empirical studies describe low levels of participation of children and adolescents in this type of education and in research in this field. Nevertheless, there are few studies that measure participation, being limited to describing case studies. In order to clarify this, a systematic bibliographic review of environmental education research written in Spanish between 1999 and 2019 was conducted, in which children and adolescents participated with different degrees of involvement. Their levels of participation were coded using Roger Hart's ladder of child participation, which consists of eight different rungs. This was correlated by statistical analysis and non-parametric tests with the year and country of publication, the type of education, and the methodology used. The results indicate a progressive increase in the levels of child and adolescent participation from 1999 to date, as well as higher levels in non-formal environmental education if qualitative methodologies are used for research (based on the work of authors from Argentina and Cuba). The importance of rethinking the place of children and adolescents in the development of environmental education and research is discussed.
\end{abstract}

Keywords: adolescent participation, child participation, environmental education research, environmental education, systematized bibliographic review.

\section{Introducción}

En los últimos ańos organizaciones internacionales como el Fondo de las Naciones Unidas para la Infancia, Unicef, y la Red Internacional de Investigación Childwatch han hecho un llamado a fortalecer los estándares de participación de la investigación con niños, niñas, adolescentes (en adelante NNA) y jóvenes (Ceballos-López y Saiz-Linares, 2019). Pese a ello, en los procesos investigativos el protagonismo de este segmento es incipiente, siendo más bien costumbre la exclusión de este grupo etario (Graham, Powell \& Taylor, 2015; Powell, Graham, Taylor, Newell \& Fitzgerald, 2011). Esta situación es crítica en naciones de bajo y mediano ingreso como las hispanoamericanas, donde producto de su cultura y su mirada de la infancia y la juventud, los niveles y calidad de participación en la investigación educativa son más bien bajos (Navas, Martínez, Valdebenito y Castillo, 2018).

Destacados líderes juveniles de Hispanoamérica como Patricia Ramos (España), Dominga Espiñeira (Chile), Militza Lízbeth Flaco Suira (Panamá), Bruno Rodríguez (Argentina), Jamie Margolin (Colombia) o María José Bejarano (Bolivia), han querido señalar que su lugar no es equiparable al de sus antecesores, puesto que gran parte de la responsabilidad de la problemática es de los adultos, pero buena parte de la propuestas de las soluciones proviene de los jóvenes y las nuevas generaciones, lo que no se condice con las responsabilidades políticas que se les han otorgado (Liebel y Gaitán, 2019).

En este sentido, los NNA alegan que han quedado excluidos de uno de los desafíos más grandes de la humanidad, pese a estar muchos de ellos y ellas más conscientes y preparados al respecto que los mismos adultos que los segregan (Lawson et al., 2019). Por este motivo, señalan energéticamente que no pueden seguir soportando el no ser oídos. Ante esto, ciertos investigadores y tomadores de decisiones (Hart, 2013; Liebel, 2007) se han interesado por la participación infantil y adolescente, preguntándose por el grado de involucramiento de los NNA en los programas, sistemas y proyectos medioambientales (Green, 2015; Imhoff y Brussino, 2013). 
Esto ha permitido entender la participación infantil y adolescente como un constructo polisémico y un proceso complejo, que involucra la transformación de las condiciones sociales en las que se desenvuelven los NNA (Susinos-Rada y Ceballos-López, 2012; Susinos-Rada, Ceballos-López, Saiz-Linares y Ruiz-López, 2019). En este sentido, la participación es tanto condición de acción de una subjetividad, como también posibilidad de construir espacios y sociedades más democráticas (Fielding, 2012). Desde esta noción de participación, académicos como Hart (1992; 2013), Robottom (1993) y Robottom y Sauvé (2003) se han interesado particularmente por entender el involucramiento de jóvenes, infantes y adolescentes en proyectos sociales, educativos o investigativos de carácter ambiental.

Esta empresa sin duda alguna tiene motivos suficientes para ser considerada: en primer lugar, la participación de la infancia y la juventud es un derecho internacionalmente establecido y se debe velar por él; en segundo término, la inclusión de estos sujetos sociales potencia los programas e instancias de las que forman parte, como también su capacidad transformadora; tercero, dicha participación trae consigo una serie de positivos efectos en las personas pertenecientes a las comunidades educativas y a los proyectos educacionales que la propician; y cuarto, se ofrece como una excelente herramienta para favorecer la formación ciudadana y la responsabilidad social con el medio ambiente (García-Pérez y de Alba-Fernández, 2012; Mager \& Nowak, 2012; Moreno-Fernández y García-Pérez, 2013; Trilla y Novella, 2011).

En este contexto, la presente investigación buscó describir los diversos niveles de participación de NNA en la educación ambiental (en adelante EA) y los grados de protagonismo que coexisten y se diferencian entre sí. Para esto, se presentan algunos antecedentes respecto de la participación de NNA en la EA, como también de los estudios que surgen a partir de este campo. Posteriormente, se ofrece una revisión bibliográfica sistematizada basada en el trabajo de Prosser y Romo-Medina (2019) y realizada a partir de estudios de EA escritos en espańol en los últimos 20 años, haciendo un descripción de los diversos niveles de participación que se encuentran allí expuestos.

\section{Participación de NNA en la EA}

En el caso específico de la EA, la participación de este segmento ha sido destacada al punto de ser considerada una característica esencial del campo de estudio (Espejel-Rodríguez y Flores-Hernández, 2012; Tréllez, 2015). Esto ha sido matizado por investigaciones empíricas, las que advierten una participación poco protagonista por parte de los NNA en el desarrollo de las actividades educativas ambientales (Calvente, Kharrazi, Kudo, \& Savaget, 2018; Matos-De Rojas, Pasek-De Pinto, Peña-Briceño y Briceño, 2018). Así planteada, la EA tiene un doble desafío: por un lado, contribuir al abandono del "antropocentrismo" y las lógicas de producción/consumo capitalistas; y por otro, trabajar las formas de participación adultocéntricas en educación (Bywater, 2014; LayLisboa y Montañes, 2018), las que dificultan el verdadero involucramiento de los NNA en los proyectos y acciones.

Por lo demás, diversos autores han identificado ciertos enfoques dentro del campo de carácter cientificista y con modelos tradicionales de difusión del conocimiento respecto de los problemas y ciclos ambientales, reduciendo la comprensión de estos fenómenos a aspectos técnicos y contables, en términos de desarrollo sostenible (Jensen, 2002; Reid, Jensen, Nikel \& Simovska, 2008). Existe también una serie de experiencias educativas contextualizadas y críticas orientadas al cambio y la transformación, las cuales buscan ampliar la visión ambiental hacia términos locales de gobernanza, cambio climático, gestión inclusiva del riesgo y desastres, seguridad y soberanía alimentaria o gestión ambiental comunitaria en general, por mencionar algunos (González-Gaudiano y Meira-Cartea, 2020; Moreno-Fernández y García-Pérez, 2013). Ante esto ha surgido también otro desafío: promover iniciativas participativas en la educación, ofreciendo espacios donde se considere la experiencia y opinión de los NNA para la construcción y gestión del conocimiento (Jensen y Schnack, 2006). 
Aquí cabría mencionar los programas educativos internacionales basados en un modelo que promueve la participación activa de los NNA. Entre estos se encuentran las Ecoescuelas, la Bandera Azul, la Green Flag y la Llave Verde, (Moreno-Fernández y Navarro-Díaz, 2015; Perales-Palacios, Burgos-Peredo, y GutiérrezPérez, 2014); los Young reporters for the environment o el programa Learning About Forests (LEAF) de la Foundation for Environmental education (De Angelis, 2016). Por último, desde una visión histórica, cabe destacar el aporte de las Agendas 21 para el desarrollo de programas participativos de EA de carácter local (PozoLlorente, Gutiérrez-Pérez \& Poza-Vilches, 2019).

Esto también ha sido un tema relevante en los diálogos entre académicos y profesionales investigadores del campo. Un buen ejemplo de esto es el 13th Invitational Seminar on Environmental Education Research, sucedido en 2015 en Bertioga, Brasil. Dicha ocasión sirvió para pensar los aportes de la pedagogía de Paulo Freire a la EA y a la investigación en EA (IEA), destacándose la necesidad de construir la investigación educativa en contextos locales y desde quienes participan en ella (Hart, Hart, Aguayo \& Thiemann, 2018); darle una mayor importancia a los afectos y a la estética (Payne, 2018); y tener una visión descolonizadora del saber ambiental que surge de los procesos de producción de conocimiento (Payne, 2018).

\section{Participación de NNA en la IEA}

Actualmente, la IEA es entendida como una investigación educativa que tiene su foco en las relaciones entre aspectos ambientales y educativos, ya sea en términos institucionales, pedagógicos, didácticos o personales, entre otros (Briggs, Trautmann \& Phillips, 2019). Esta por tanto, se desarrolla en escenarios formales y no formales en donde los saberes pedagógicos confluyen con saberes sociales, populares, indígenas y ambientales (Caride Gómez, 2008). En este campo, se debieran considerar las particularidades de los contextos sociales y las realidades ecológico-culturales en que se sitúan y relacionan las distintas personas, a la vez que se develan de manera colectiva las causas y posibles consecuencias de determinados problemas ambientales (Calixto-Flores, 2012).

Algunos de los aportes y contribuciones más significativos que ofrece la IEA según Benayas, Gutiérrez y Hernández (2003), es que sirve de instrumento para la profesionalización, reconocimiento, legitimación del sector y para la discriminación y control de la calidad de la planificación y diseńo de programas en que se debe diferenciar entre prácticas eficaces y fallidas. También sirve como reporte de transparencia y equidad, junto con las implicancias políticas y sociales que derivan de la evidencia obtenida. Por último, puede servir para la reflexión, análisis, empoderamiento, activismo y cambio socioambiental de profesionales, educandos y comunidades (Benayas et al., 2003).

Revisiones en español de la disciplina han permitido establecer que España, Venezuela, México y Colombia son los países en los cuales se han desarrollado más investigaciones; la Revista Luna Azul es el lugar donde más se ha publicado respecto al tema; y que, a partir del 2006, incrementó el número de estas. Aun así, desde los mismos investigadores que han realizado revisiones bibliométricas en el campo, se señala la importancia de cuestionarse las relaciones que tiene la EA y la IEA con ciertos grupos de sujetos (González-Gaudiano y Arias-Ortega, 2015; Medina-Arboleda y Páramo, 2014; Prosser y Romo-Medina, 2019; Sepúlveda, 2015). Esto, para el caso de los NNA, fue abordado por parte de la investigación de Martínez-Agut, Ull \& AznarMinguet (2014), la cual analiza los documentos oficiales de España que reglarían el desarrollo de la Early Childhood Education For Sustainability (ECES).

Los autores señalan que, en general, se ofrecen actividades y metodologías a aplicar con los NNA, pero que se encuentran pocos criterios entre las normativas para aquellos profesionales e instituciones que busquen promover la ECES, lo que trae consigo una dificultad para desarrollar las competencias necesarias para su 
correcta implementación (Martínez-Agut et al., 2014). Sobre la base de esta afirmación se podría pensar que el protagonismo de NNA es un elemento implícito de la EA, pero que ha sido escasamente incentivado y evaluado, como tantos otros temas en la disciplina ${ }^{1}$.

El lugar que puede jugar ahí la IEA es fundamental, pues procesos de este tipo pueden significar verdaderas revoluciones participativas en la escuela, que involucren altos grados de protagonismo infantil y adolescente, o pueden ser simplemente experiencias donde un adulto aparece con un instrumento que apenas explica, que rápidamente aplica y cuyos resultados alcanzados se van a su universidad o equipo de investigación. En este escenario, los niveles de participación de NNA en IEA o estudios de EA son tan variados como existentes investigadores, equipos de investigación, escuelas, docentes, estudiantes y experiencias educativas (Ceballos-López y Saiz-Linares, 2019).

Dentro de los pocos ejercicios investigativos que han buscado develar estas particulares condiciones de participación, cabe mencionar el estudio cualitativo realizado por Prado-Fuentes y Pérez-Campusano (2011) con jóvenes universitarios de España. En este trabajo los autores quisieron analizar los niveles de participación de este grupo en el programa de gestión de residuos universitarios, identificando sus niveles de participación como "informativos" y "consultivos". En otro estudio cualitativo guiado por Matos-de Rojas et al. (2018), se realizaron entrevistas a 50 docentes de EA de Venezuela, consultándoles acerca del nivel de participación de los NNA en sus actividades. Sobre la base de los análisis realizados, se pudo determinar que solo el $17 \%$ de estos educadores promovía actividades realmente participativas.

Como ya se esbozó arriba, estos estudios de caso difícilmente son generalizables a toda la región, o inclusive a sus propios países, lo que dificulta analizar las tendencias regionales respecto del tema. Así mismo, si bien existen otras revisiones que indagan en la incorporación de NNA en la EA, estas no incorporan la dimensión de la participación como algo fundamental, limitándose a la descripción de tendencias bibliométricas o temáticas al interior del campo.

En este marco, la investigación realizada aquí profundiza la revisión bibliométrica descriptiva (RBD) realizada por Prosser y Romo-Medina (2019), diversificando las estrategias de búsqueda y de análisis de datos, con tal de examinar el nivel de participación de NNA en la IEA. En esa revisión, los autores detectaron una progresión creciente y discontinua de artículos —en su mayoría proveniente de España—, que se publican en la Revista Luna Azul y que las investigaciones son, principalmente, de tipo empírico y metodológico. A su vez, describieron nodos críticos de la EA con NNA y primera infancia, tales como las metodologías innovadoras, la predominancia de conceptos como representaciones sociales o actitudes, el uso didáctico de los animales o la educación ambiental comunitaria. Dicho estudio, si bien fue un aporte al campo, no permitió analizar los diferentes niveles de participación de los NNA en los estudios de EA, motivo por el cual se creyó necesario generar un nuevo proceso investigativo.

También cabe mencionar que la RBD anterior se sustentaba en una línea nórdica-sajona de la EA, la cual está orientada a entender las particularidades del campo en el trabajo con NNA y, por sobre todo, con primera infancia (Davis \& Elliot, 2014), mientras que el presente trabajo está orientado a describir los niveles de participación en la literatura, siguiendo más bien una línea de desarrollo propia de Espańa, Latinoamérica y el Caribe (Calixto-Flores, 2012; Caride Gómez, 2008).

1. Por ejemplo, la educación continua del docente, el rescate de los saberes populares y tradicionales, la dinamización de la comunidad educativa hacia un cambio sustentable y que trascienda el establecimiento educacional, el ecofeminismo y la perspectiva de género en la EA, como también la inclusión de las necesidades, intereses y particularidades del territorio y comunidades locales. 
Esto es fundamental en Hispanoamérica, pues los representantes de la región han señalado que la participación es un principio basal de la EA (Calixto-Flores, 2010; García-Pérez y de Alba-Fernández, 2012; González-Gaudiano y Arias-Ortega, 2015; Tréllez, 2015). Por este motivo, es relevante desarrollar un ejercicio investigativo que refleje los diversos niveles de participación de los NNA en la EA y sus estudios, de modo de exponer tanto las experiencias con una clara tendencia participativa, como aquellas otras en las cuales las nuevas generaciones son usuarias o receptoras.

Por tanto, el objetivo general del estudio fue identificar los niveles de participación de los NNA en los artículos publicados en revistas especializadas y bases de datos iberoamericanas en los últimos 20 años. Para esto, se utilizó la escalera de participación de Roger Hart (1992), buscando describir diferentes grados de involucramiento de los NNA en los estudios y/o experiencias educativas, pudiendo tratarse de coinvestigadores y protagonistas activos a meros objetos de estudio de ellas. El objetivo específico fue distinguir los diversos niveles de participación por medio del análisis de una serie de variables bibliométricas de interés, las que fueron correlacionadas con los escalones sugeridos por Hart (1992).

\section{Metodología}

En la presente investigación se realizó una revisión bibliográfica sistematizada (RBS). Esta metodología busca identificar tendencias y nodos críticos a partir de una serie de criterios que permiten trabajar con antecedentes bibliográficos acumulados por investigaciones previas (Grant \& Booth, 2009). Es utilizada por su sistematicidad a la hora de hacer análisis de los principales problemas de un ámbito, para explorar áreas de conocimiento incipientes, y también para detectar estados del arte, nuevas tendencias y oportunidades de investigación (Booth, Sutton \& Papaioannou, 2016).

Durante el desarrollo de esta investigación se siguieron las recomendaciones de diversos autores (García-Vinuesa y Meira-Cartea, 2019; Manchado-Garabito, Tamames-Gómez, López-González, Mohedano-Macías y Veiga de Cabo, 2009; Sánchez-Meca y Botella, 2010) tanto para la realización como para la descripción del método, en especial para la elaboración de la estrategia de búsqueda, los criterios de inclusión y exclusión, la definición de la variable de estudio (niveles de participación infantil y adolescente) y para el proceso mismo de revisión y extracción de los datos.

\section{Estrategia de búsqueda}

Se realizó una búsqueda de aquellos artículos publicados en las bases de datos SciELO, Dialnet, Redalyc y JSTOR y en el Repositorio Digital de la Universidad de Chile. Adicionalmente se revisaron todos los números de la Revista Luna Azul de la Universidad de Caldas, publicación hispanohablante especializada en temas medioambientales, seleccionada para el presente caso dado que revisiones anteriores la señalan como la más influyente en el campo de la EA (Medina-Arboleda y Páramo, 2014; Prosser y Romo-Medina, 2019; Sepúlveda, 2015). En todos estos espacios académicos se ingresó el siguiente código booleano:

Infancia OR Juventud OR Niñez OR Adolescencia OR Infante OR Joven OR Niño OR Niña OR Adolescente AND

Educación ambiental OR Educación para el desarrollo sostenible OR Educación para la sustentabilidad. 
De manera adicional, y para sumar rigurosidad en la búsqueda, se rastrearon otras investigaciones mediante la introducción de una serie de palabras clave, pertenecientes a diversos tesauros, a saber: infancia, niñez, adolescencia, juventud, educación ambiental, educación para el desarrollo sostenible, educación para la sustentabilidad, sostenibilidad y ambiental ${ }^{2}$.

Para este proceso, los autores utilizaron la misma sintaxis de búsqueda y un grupo importante de artículos de una RBD anterior (Prosser y Romo-Medina, 2019), lo que sumado a las nuevas estrategias de búsqueda y objetivos de estudio tuvo por consecuencia diferencias tanto en el número de trabajos identificados e incluidos, como en los análisis que se despliegan a lo largo de las metodologías y resultados.

\section{Criterios de inclusión}

La selección de los estudios fue realizada de manera conjunta por los tres autores, teniendo como condición el consenso en el cumplimiento de los criterios de inclusión para cada uno de los estudios seleccionados. La valoración de este cumplimiento fue dicotómica y cualitativa, esto es, que los trabajos se clasificaron en "aceptado" o "rechazado", de acuerdo con la presencia de los siguientes criterios:

1. Investigaciones empíricas de la disciplina y/o disciplinas afines difundidas mediante comunicaciones científicas.

2. Publicados en español en los últimos 20 años (de 1999 hasta el 14 de febrero de 2019). Lo que se buscó con este filtro fue acceder al campo de estudio desarrollado en Hispanoamérica.

3. Publicados en revistas científicas con un sistema de revisión por pares (peer review) que garantice la calidad de esta (Liberati et al., 2009).

4. Estudios empíricos que consideraran como participantes a NNA, incluidos aquellos jóvenes que aún pertenecían al sistema escolar.

Se confeccionó una matriz de registro bibliográfico en la que se fueron depositando los artículos que cumplieron con los criterios antes mencionados. Los datos del estudio se distribuyeron a lo largo de diversas columnas de una planilla de Microsoft Office Excel, las que se encontraban tituladas bajo los siguientes rótulos: autores, título del artículo, año de publicación, nombre, URL o doi —en caso de que existiera一, revista, país, número de muestra, edad de la muestra, metodología de la investigación, citaciones, ámbito de la educación, enfoque de EA, resumen y las palabras clave. En la planilla, se destinó una columna para designar el nivel de participación asignado a cada estudio, basándose en la taxonomía utilizada para el análisis.

\section{Criterios de exclusión}

Se excluyeron artículos en dos momentos: en el primero, se descartaron las investigaciones en las que no había información suficiente para determinar la participación efectiva de los NNA en ellas, como también se rechazaron aquellos artículos en los que, si bien el objeto de estudio eran los NNA, las metodologías se aplicaron a personas

2. La introducción de estos términos responde a que la EA recibe diferentes nombres y tratamientos en Hispanoamérica según la posición de cada autor; misma situación sucede con los conceptos de infancia y juventud. Para ahondar en diferentes posiciones que coexisten también en la educación para el cambio climático (EpCC), se recomienda ver el estudio de González-Gaudiano y Meira-Cartea (2020) y la revisión de investigaciones con estudiantes secundarios de García-Vinuesa y Meira-Cartea (2019). 
adultas. En segunda instancia, se excluyeron aquellos que fueran revisiones o estudios instrumentales. Para realizar ambas exclusiones se puso especial atención en el apartado de metodología de los textos, identificando la mención (o no) de sus participantes y la referencia a las técnicas de producción y análisis de la información.

Para graficar el proceso se realizó un diagrama de flujo (ver Figura 1). Al comienzo del procedimiento se agregó el código booleano en los motores de búsqueda y se identificaron 4.424 trabajos. A partir de esto, se seleccionaron 205 artículos que cumplieron con los criterios de inclusión y, posteriormente, se realizó la primera de las exclusiones, con lo cual quedó un total de 180 artículos. Finalmente, se optó por dejar fuera aquellos estudios instrumentales y revisiones, debido a la evidente baja participación de los NNA en estos, y con el fin de reducir el sesgo en los resultados; con ello, el corpus final quedó compuesto por 119 estudios empíricos.

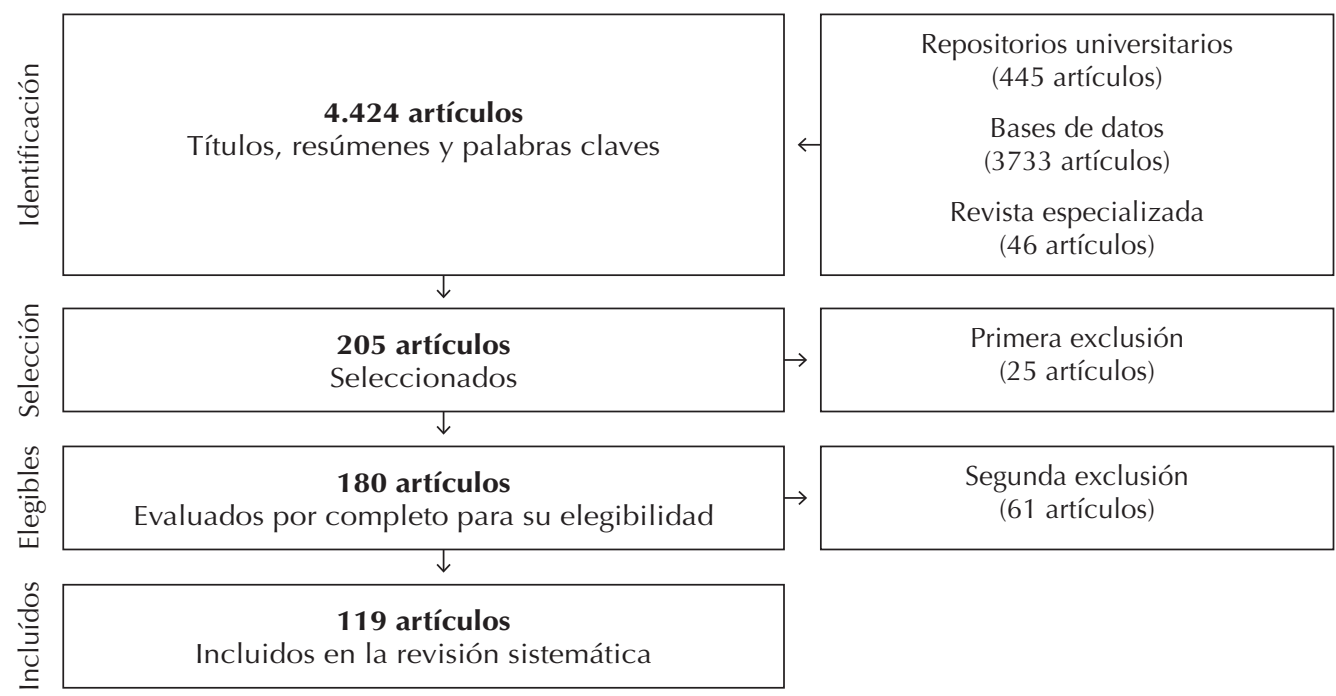

Figura 1. Diagrama de flujo de la revisión bibliográfica sistematizada.

Fuente: Elaboración propia basado en declaración PRISMA (Liberati et al., 2009).

\section{Codificación}

Los artículos seleccionados fueron repartidos en igual número entre los tres autores, luego de lo cual se realizó — de manera independiente y separada - una serie de lecturas superficiales para establecer las clasificaciones de los trabajos. Se discutió de manera conjunta acerca de aquellos estudios que generaron dudas o en los que los otros autores disintieran de la valoración ofrecida por el primer lector. Para la valoración definitiva de los trabajos se cruzaron todas las lecturas y se tuvo como criterio de confiabilidad el que el consenso fuera de un $100 \%$. A continuación, se describen las variables de interés del presente estudio.

Nivel de participación infantil y adolescente en EA. En esta investigación se entendió este constructo como el grado de involucramiento y protagonismo de uno o varios NNA en temáticas, actividades y proyectos ambientales que les competen en diversas esferas de su vida (Susinos-Rada y Ceballos-López, 2012). Al respecto, si bien existen diversas propuestas para graduar la participación de NNA (e.g., Trilla y Novella, 2011), se decidió utilizar la escalera de participación de Roger Hart (1992) por su extendido reconocimiento en la EA (Davis, 2005; Læssøe, 2010; Prado-Fuentes y Pérez-Campusano, 2011) y por la claridad operacional de los ocho niveles planteados (ver Tabla 1). 
Tabla 1

Niveles de participación de la taxonomía de Roger Hart (1992)

\begin{tabular}{|c|c|c|}
\hline Categoría & Definición & Puntaje \\
\hline $\begin{array}{l}\text { Iniciados por los niños, } \\
\text { decisiones compartidas con los } \\
\text { adultos }\end{array}$ & $\begin{array}{l}\text { Consiste en la inclusión de la participación de personas } \\
\text { adultas en procesos iniciados desde la infancia o } \\
\text { adolescencia. }\end{array}$ & 8 \\
\hline $\begin{array}{l}\text { Iniciada y dirigida por los } \\
\text { niños }\end{array}$ & $\begin{array}{l}\text { Este nivel hace referencia a cuando los NNA deciden, } \\
\text { de manera autónoma, iniciar y ejecutar un proceso } \\
\text { investigativo sin la participación directiva de personas } \\
\text { adultas. }\end{array}$ & 7 \\
\hline $\begin{array}{l}\text { Iniciada por los adultos, } \\
\text { decisiones compartidas con los } \\
\text { niños }\end{array}$ & $\begin{array}{l}\text { Alude a cuando los adultos deciden, una vez iniciado } \\
\text { el proyecto, compartir las decisiones respecto de su } \\
\text { desarrollo con las NNA. }\end{array}$ & 6 \\
\hline Consultados e informados & $\begin{array}{l}\text { Dice relación a cuando el proyecto es iniciado y } \\
\text { dirigido por los adultos y los NNA cumplen la función } \\
\text { de consultores, comprendiendo el proceso y siendo } \\
\text { consideradas importantes sus opiniones. }\end{array}$ & 5 \\
\hline Asignado pero informado & $\begin{array}{l}\text { Alude a cuando los NNA se ofrecen como voluntarios } \\
\text { en un proyecto iniciado por adultos, comprenden sus } \\
\text { intenciones y el rol significativo que desempeñan en él. }\end{array}$ & 4 \\
\hline Participación simbólica & $\begin{array}{l}\text { Apunta a aquellos casos en los que aparentemente } \\
\text { los NNA tienen la posibilidad de expresarse, cuando } \\
\text { en realidad tienen una baja o nula influencia en el } \\
\text { desarrollo del proyecto investigativo. }\end{array}$ & 3 \\
\hline Decoración & $\begin{array}{l}\text { Refiere a aquellas ocasiones en que los NNA no tienen } \\
\text { idea alguna en qué consiste el proyecto, ni tampoco } \\
\text { posibilidad de incidir en él, por lo que hacen tan solo } \\
\text { un acto de presencia. }\end{array}$ & 2 \\
\hline Manipulación & $\begin{array}{l}\text { Alude a aquellos casos en que los NNA no comprenden } \\
\text { el objetivo de la investigación ni sus acciones en ella. } \\
\text { Por tanto, los adultos requieren de su participación con } \\
\text { fines instrumentales. }\end{array}$ & 1 \\
\hline
\end{tabular}

Fuente: Elaboración propia.

Para identificar a qué nivel correspondía cada uno de los artículos, se desarrolló un árbol de decisiones que constó de seis indicadores (ver Figura 2): el primero de estos revisaba si los participantes comprendían el objetivo del estudio o acción; el segundo, si entendían y si se les había explicado su rol en él; el tercero, si su papel era significativo o más bien simbólico; el cuarto, si su opinión era relevante o no; el quinto, si el proceso era iniciado por adultos o por NNA, distinguiendo en el primer caso si es que, una vez iniciada la investigación, consultaban opiniones o decidían en conjunto con los NNA; y el sexto, en aquellos casos que iniciaban los NNA, si estos ejecutaban solos o con adultos. 


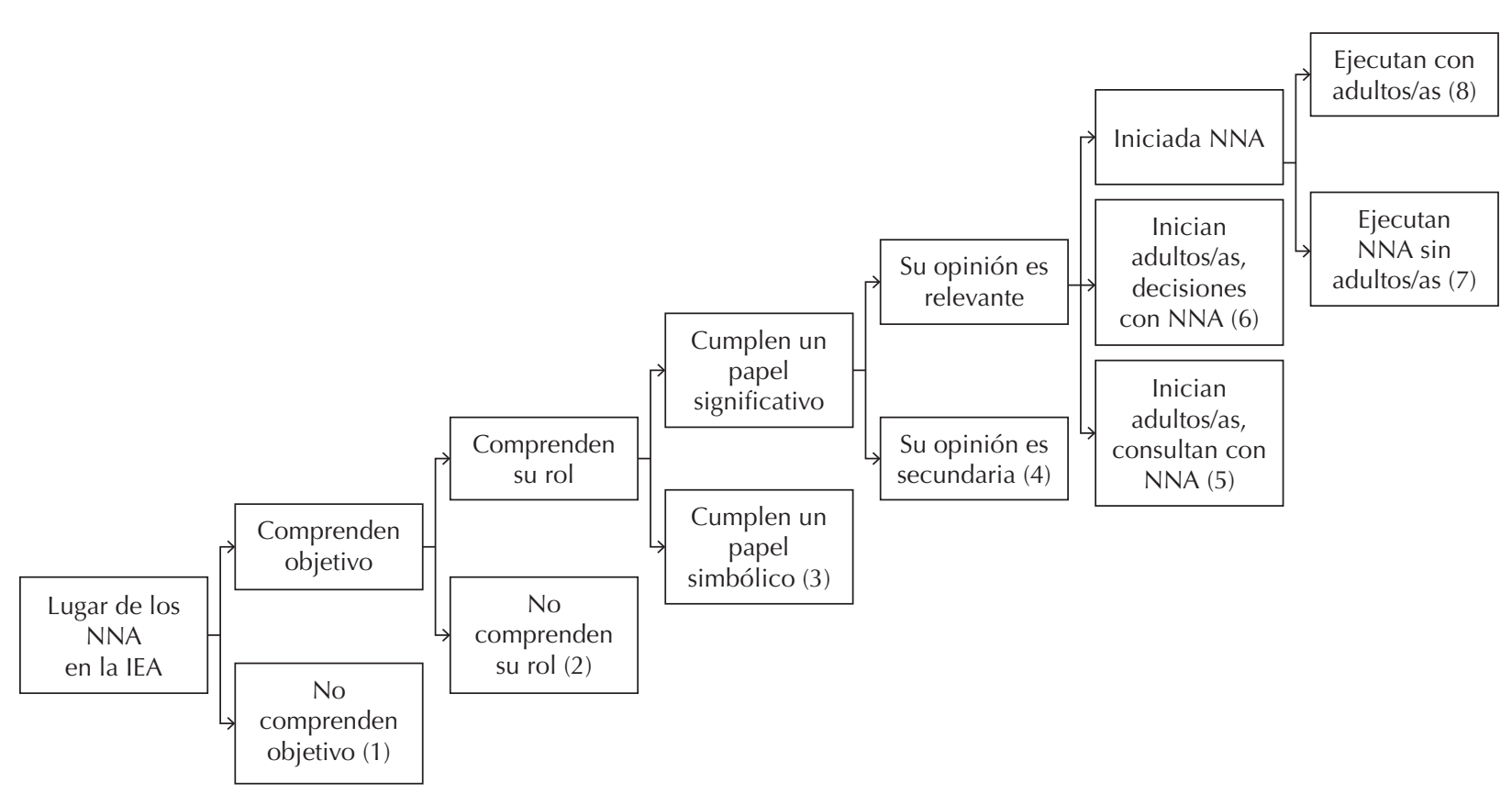

Figura 2. Árbol de decisiones para establecer los niveles de participación .

Fuente: Elaboración propia.

Año y país. Se consideró para cada uno de los artículos su año de publicación y el país de realización de la investigación en EA.

Tipos de educación. Utilizando la definición de María Novo (1996), se distinguió entre "la educación ambiental que se desarrolla en ámbitos escolares (educación formal) con aquella otra que vienen realizando las organizaciones no gubernamentales, grupos ecologistas, ayuntamientos y comunidades autónomas (educación no formal)” (p. 75). Se habló, por tanto, de una educación ambiental formal (EAF) y de una educación ambiental no formal (EANF).

Enfoque metodológico. Sobre la base de la revisión realizada por Green (2015), se distinguieron tres enfoques metodológicos. En primer lugar, la metodología cuantitativa con un marcado enfoque positivista orientado a la contrastación de hipótesis, mediante la medición de variables que se recogen a partir de instrumentos estandarizados, a los que finalmente se le extraen datos numéricos para la realización de análisis estadísticos. En segundo lugar, la metodología cualitativa orientada a la comprensión profunda de fenómenos que investiga mediante enfoques interpretativos, descriptivos, narrativos (por ejemplo, escritos, verbales, visuales, etc.) y etnográficos, entre otros. Por último, la metodología mixta, en la cual la recolección y análisis de los datos se realiza de manera cuantitativa y cualitativa, pudiendo realizarse de forma secuencial, paralela o mezclada.

\section{Análisis}

Dado el carácter descriptivo de esta revisión y la utilización del recuento de votos como estrategia de medida, para examinar los datos se realizaron análisis estadísticos de heterogeneidad para cada una de las variables y se estudió su nivel de significancia según la prueba de Chi cuadrado (Franke, Ho \& Christie, 2017). Luego, se comparó el nivel de participación de acuerdo con las distintas variables consideradas en el estudio a través de la prueba no paramétrica Kruskal-Wallis (K-W), que toma en cuenta rangos promedio (rp) de variables ordinales. Estos análisis fueron incorporados con la intención de robustecer los resultados e ir más allá de la mera presentación descriptiva, como es usual en este tipo de revisiones (Ardoin \& Bowers, 2020). 


\section{Resultados}

\section{Caracterización de los artículos}

En la Tabla 2 se muestran las características de las 119 IEA que fueron consideradas para esta investigación. En ella se puede observar un aumento sostenido y significativo ( del número de publicaciones en los últimos 20 años, incrementándose en promedio 5,95 artículos anualmente.

Respecto de los países, se puede observar que tanto Espańa (21,8\%) como Colombia (21\%) y México (19,3\%) lideran la producción de IEA. Los países con menos producción son Ecuador, Paraguay, Puerto Rico y Brasil con solo un artículo publicado. En cuanto a esta última nación, es importante considerar que esta revisión solo incluyó artículos en español, por lo que esta cifra puede estar subestimada.

Así mismo, se pudo encontrar que las investigaciones reportadas en estos artículos en su mayoría tratan de EAF (71\%) que utilizan una metodología de corte cualitativo (52\%). Finalmente, las categorías en cada una de estas variables muestrean diferencias significativas según la prueba de Chi cuadrado.

Tabla 2

Caracterización de artículos de EA durante los últimos 20 años $(n=119)$

\begin{tabular}{|c|c|c|c|c|c|c|c|}
\hline Variable & $\mathrm{N}$ & $\%$ & $\mathrm{X}_{(\mathrm{gl})}^{2}$ & Variable & $\mathrm{n}$ & $\%$ & $\mathrm{X}_{(\mathrm{gl})}^{2}$ \\
\hline Año de publicación & & & & Ámbito de la EA & & & \multirow{4}{*}{$21,186_{(1)}{ }^{* *}$} \\
\hline $1999-2003$ & 3 & $3 \%$ & \multirow{4}{*}{$71,151_{(3)}{ }^{* *}$} & EA formal & 85 & $71 \%$ & \\
\hline $2004-2008$ & 16 & $13 \%$ & & EA no formal & 34 & $28 \%$ & \\
\hline $2009-2013$ & 36 & $30 \%$ & & & & & \\
\hline 2014-2018 & 64 & $54 \%$ & & & & & \multirow{5}{*}{$19,479_{(2)}^{* *}$} \\
\hline Pais & & & & Metodología & & & \\
\hline España & 27 & $22,7 \%$ & \multirow{10}{*}{$133,588_{(13)}{ }^{* *}$} & Cualitativa & 62 & $52 \%$ & \\
\hline Colombia & 25 & $21,0 \%$ & & Mixta & 32 & $27 \%$ & \\
\hline México & 23 & $19,3 \%$ & & Cuantitativa & 25 & $21 \%$ & \\
\hline Chile & 10 & $8,4 \%$ & & & & & \\
\hline Cuba & 9 & $7,6 \%$ & & & & & \\
\hline Venezuela & 9 & $7,6 \%$ & & & & & \\
\hline Costa Rica & 5 & $4,2 \%$ & & & & & \\
\hline Perú & 4 & $3,4 \%$ & & & & & \\
\hline Argentina & 3 & $2,5 \%$ & & & & & \\
\hline Otros países & 4 & $<1 \%$ & & & & & \\
\hline
\end{tabular}




\section{Niveles de participación}

Utilizando la taxonomía de Hart (1992; 2013) se encontró que ninguno de los 119 artículos analizados describía un nivel de participación inferior a tres puntos (manipulación y decoración) ni superior a seis (iniciada y dirigida por NNA y proyectos iniciados por menores y compartidos con adultos). De los niveles de participación hallados, la asignación e información se encuentra presente en el 41,2\% de los artículos, seguida por la participación simbólica $(28,6 \%)$, la consulta e información $(21,8 \%)$ y los proyectos iniciados por adultos, pero compartidos con NNA $(8,4 \%)$.

\section{Niveles de participación de acuerdo con las variables de estudio}

Como se puede observar en la Figura 3, el grado de diversificación de la participación en los artículos de EA aumentó en los últimos cuatro años. Al comienzo de la década de 2000 predominaba la participación simbólica, mientras que actualmente el nivel asignados pero informados es el más reiterado, apareciendo recién a partir del 2008 también el nivel de iniciados por los adultos pero compartidas las decisiones con los NNA.

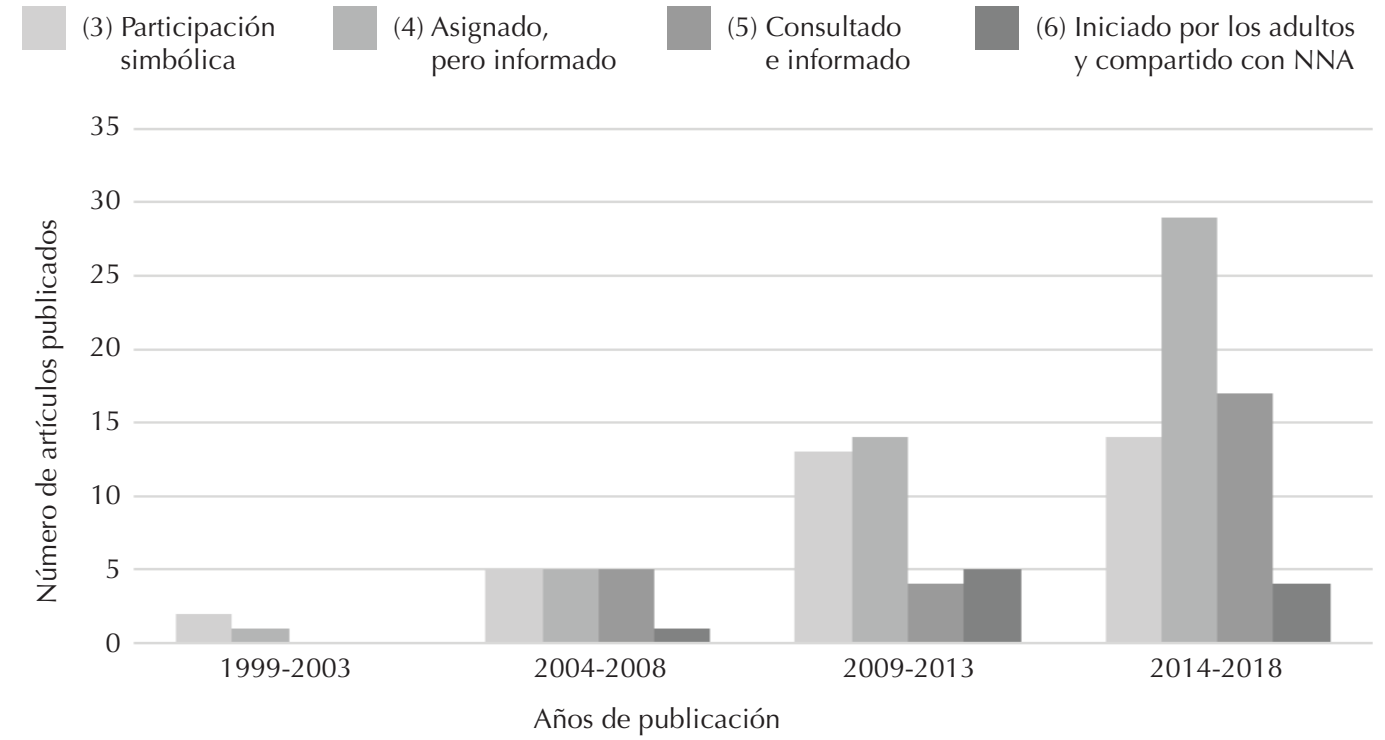

Figura 3. Niveles de participación en la IEA en los últimos 20 años.

Fuente: Elaboración propia.

Para analizar la relación del nivel de participación según país, se elaboró un ranking de valores continuos según los niveles de participación en la escala de Hart (1992; 2013), ubicando en el extremo inferior investigaciones con ausencia de participación infantil y adolescente (puntuación 0) y en el superior el nivel máximo (puntuación 1).

Se detectó que, en general, los países tienen un nivel de participación de 0,44 ( $D E=, 073)$. El país que alcanza mayor nivel de participación de NNA en EA es Argentina (0,52), aunque solo con tres artículos, y aquellos con menor participación son Ecuador, Paraguay y Puerto Rico, todos con un índice de 0,29. También cabe considerar que países como Espańa, Colombia y México presentan la mayor cantidad de artículos y niveles de participación superiores a la media. Esto se puede observar en la Figura 4, la cual solo presenta países de Hispanoamérica que hayan cumplido con la totalidad de criterios de la revisión. 


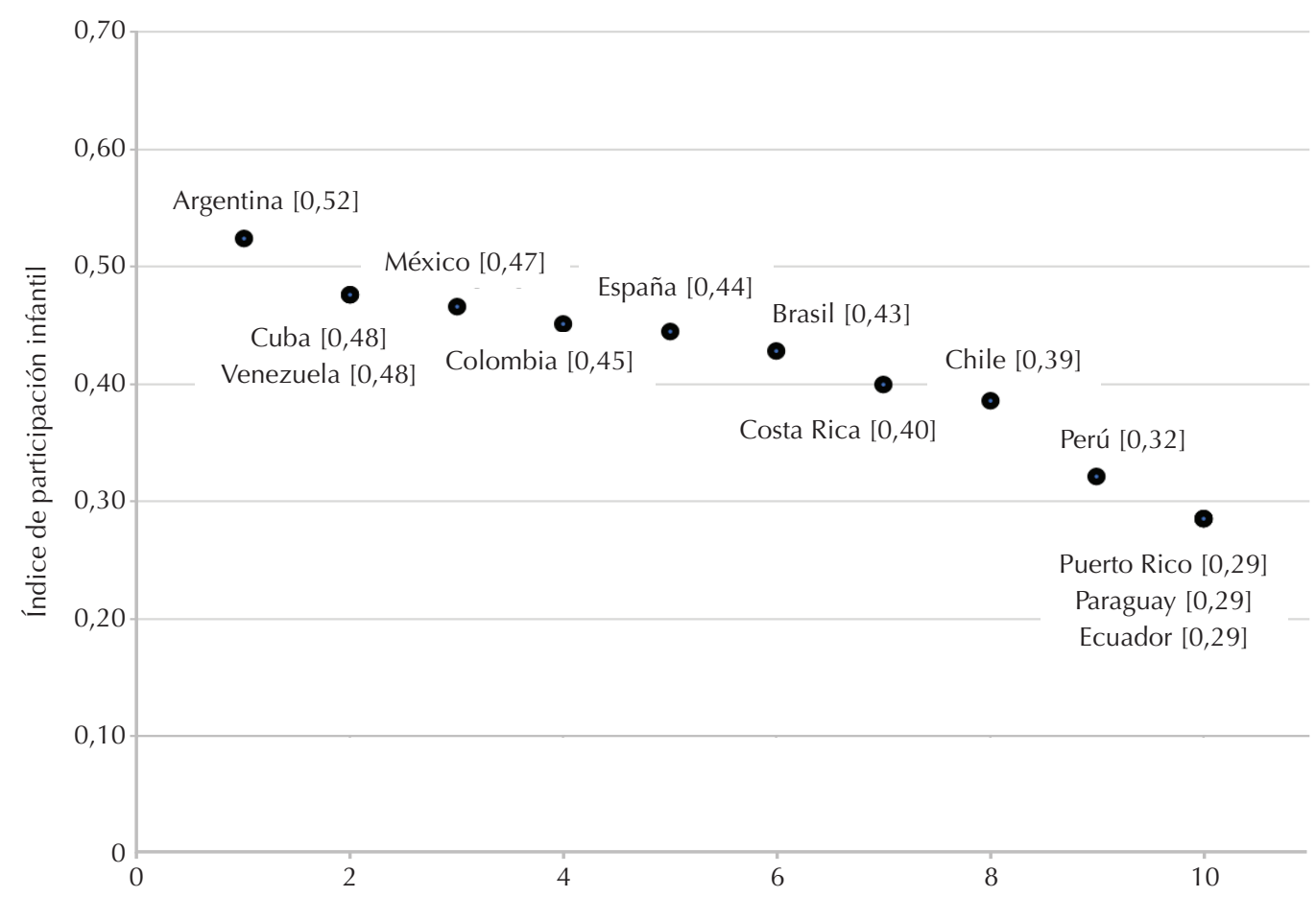

Figura 4. Niveles de participación en los últimos 20 años según país. Entre paréntesis el promedio de participación por país.

Fuente: Elaboración propia.

Se compararon los niveles de participación de los artículos según ámbito y metodología de IEA, encontrando diferencias significativas en todas las variables analizadas. En cuanto al ámbito, se halló que la participación ( es mayor en estudios de EANF ( $\mathrm{rp}=79,29)$. A su vez, en relación con el enfoque metodológico, los estudios cualitativos fomentan una mayor participación ( $\mathrm{rp}=71,85)$, que los métodos mixtos $(\mathrm{rp}=58,56)$ y los cuantitativos $(\mathrm{rp}=32,44)$.

\section{Discusión final}

La presente revisión buscó medir el nivel de participación de los NNA en los estudios de EA publicados en español en los últimos 20 años. Este ejercicio se realizó para ofrecer un panorama global respecto de la participación en los procesos investigativos de EA. A esto se suma que el involucramiento es un derecho de los NNA, que moviliza procesos de transformación de las instancias donde estos participan, que genera una serie de efectos positivos en las personas y comunidades, y que es una herramienta solvente para propiciar el desarrollo de la ecociudadanía (Mager \& Nowak, 2012; Trilla y Novella, 2011).

En este sentido, este trabajo sirve para alertar a los profesionales que se paran desde este campo, puesto que dada la magnitud de los desafíos del presente es necesario que la EA consolide a la educación e investigación participativa como sus bases efectivas de desarrollo disciplinar (Briggs et al., 2019; García-Pérez y de AlbaFernández, 2012). Por esto, los procesos de (co)educación y de investigación con potenciales ecociudadanos no deben basarse en la mera transferencia de los conocimientos y problemas ambientales, en la tecnocracia adulto céntrica de la gestión de estas iniciativas, ni tampoco en la retórica de la inmadurez para excluir a los NNA de ciertas fases (Jensen, 2002; Jensen \& Schnack, 2006; Le Grange, 2009; Moreno-Fernández y García-Pérez, 2013). 
Esto tiene particular importancia en un campo como la EA, pues precisamente lo que esta busca es cuestionar la red de relaciones que se construyen socialmente con la biosfera y los medios considerados naturales, siendo fundamental desde una perspectiva de la participación, que se cuestione por las lógicas subyacentes de poder presentes en los países, territorios y escuelas (Robottom, 1993; Robottom \& Sauvé, 2003). Cabe señalar de todas maneras que este proceso no es solo producto de un redescubrimiento y reinterpretación de un campo académico, político y, en definitiva, disciplinar, sino que también es fruto de la incesante presión social de un creciente colectivo político de jóvenes y NNA, que claman con fuerza por una mayor participación en la definición y solución de las problemáticas ambientales (Liebel y Gaitán, 2019).

En este sentido, los resultados de este estudio ofrecen un panorama de suma relevancia: aunque en menor medida, investigadores en EA han propiciado poco a poco espacios investigativos que consideran y promueven la participación de los NNA. Sin embargo, la mayoría de los estudios permanece en niveles de mera participación asignada. Lo anterior se condice con los hallazgos de otras investigaciones empíricas anteriores (Matos-de Rojas et al., 2018), las cuales inclusive coinciden en que el nivel de participación ronda entre la asignación e información y, a lo sumo, la consulta de los participantes (Prado-Fuentes y Pérez-Campuzano, 2011).

A su vez, estudios de casos y revisiones anteriormente realizadas señalan también que la EANF promueve una mayor participación (Calvente et al., 2018). Así mismo, revisiones sistemáticas acerca de participación de NNA en la educación y en la EA, han permitido constatar que su nivel es más alto en investigaciones que utilizan metodologías cualitativas (González, Hernández-Saca \& Artiles, 2017; Green, 2015).

Pese a que los resultados pudieran ser de algún modo desalentadores, cabe destacar que el interés por la participación es algo propio de la racionalidad ambiental que se ha desarrollado en España, América Latina y el Caribe (González-Gaudiano y Meira-Cartea, 2020; Tréllez, 2015) y que, por tanto, deben existir ciertas barreras o dificultades que impiden desarrollar una síntesis transformadora que nutra a los sistemas educativos y a los equipos investigativos de la región, en pos de desarrollar una mayor participación de los educandos (Calixto-Flores, 2010).

Según estudios anteriores, la cantidad de tiempo que se necesita para generar instancias participativas, la baja formación y protagonismo ciudadano de educadores e investigadores, la escasa práctica de los NNA participando y las deficientes herramientas estatales para promover la participación, se pueden configurar como obstaculizadores incluso en aquellos espacios donde se le desea fomentar (Bywater, 2014; Varela-Losada, Pérez-Rodríguez, ÁlvarezLires y Álvarez-Lires, 2014). Por este motivo, es necesario que educadores e investigadores se instruyan en estas artes y sean capaces de reflexionar y evaluar de manera crítica su propia práctica y sus relaciones de poder con los educandos (García-Pérez y de Alba-Fernández, 2012).

\section{Limitaciones}

A partir de lo antes dicho, es necesario esbozar una primera limitación evidente: en esta revisión no participó ningún NNA, ni se le pidió la opinión de cómo realizarla a ninguno de estos. Futuras investigaciones debieran seguir pensando de manera creativa el cómo propiciar cada vez más y mejores niveles de participación, inclusive en ejercicios de revisión y síntesis. Respecto de esto último, futuras revisiones sistemáticas y estudios empíricos bien podrían preguntarse y medir la "calidad" de la participación infantil y adolescente.

Otra limitación del presente estudio es que una serie de proyectos de EA comunitaria y popular se suelen transmitir y comunicar por medios diferentes al académico-racional-escrito, por lo que la inclusión de este tipo de proyectos requiere de metodologías más avanzadas y de recursos investigativos mayores (Guevara, Whelan \& Flowers, 2009). A su vez, dada la barrera idiomática del portugués, no se puede afirmar a ciencia cierta si los resultados de Brasil están correctamente estimados. En la misma senda, la exclusión de base de datos internacionales como Scopus o Clarivate puede sesgar los datos obtenidos en el presente trabajo. 
Respectos de las limitaciones metodológicas, esta revisión utilizó un criterio intencionado: la búsqueda de artículos en español publicados en revistas iberoamericanas, lo que pudo dejar fuera investigaciones realizadas en esta región, pero publicadas en inglés. Asumimos este sesgo, dado que el uso del idioma inglés no es extendido en Iberoamérica, por lo que es probable que los profesionales de la EA accedan a información a través de textos en español. Esta decisión pudo sesgar la cantidad de artículos encontrados y, por tanto, la estimación del nivel de participación por país. Futuros trabajos debieran considerar el idioma de publicación como una variable relevante y comparar si los niveles de participación reportados en los artículos varían con el idioma de publicación o incluso con el nivel de impacto de la revista donde se publica.

Por último, futuras investigaciones podrían considerar completar una matriz de información más compleja, incluyendo variables como la edad de los participantes, el tamaño de la muestra, el tiempo de ejecución de la investigación, el contenido de las actividades y el género de los investigadores. También sería interesante preguntarse por otros aspectos de la participación que transcienden la medición de niveles o grados para aportar con visiones más holísticas. Por ejemplo, sería novedoso que alguna investigación abordara el modelo de Lundy (2013) de participación indagando en los espacios, la voz, la audiencia y la influencia de los NNA en los procesos educativos ambientales.

Con todo, la presente investigación espera ser un antecedente importante a la hora de pensar la participación que hemos permitido como campo a NNA, reafirmando la relevancia que tiene el que estos sean sujetos activos de los procesos educativos ambientales en los que se involucren, en vez de ser meros receptores. Dicho esto, si la IEA sirve para el perfeccionamiento del campo, la inclusión de estos actores sociales implica no solo garantizar sus derechos, sino también contribuir a la mejora de las diversas fases de los programas e iniciativas de EA: ya no pueden ser vistos tan solo como un futuro prometedor, pues para enfrentar nuestra crisis los NNA son las personas indispensables del presente.

El artículo original fue recibido el 23 de abril de 2020

El artículo revisado fue recibido el 19 de agosto de 2020

El artículo fue aceptado el 1 de septiembre de 2020

\section{Referencias}

Ardoin, N. M. \& Bowers, A. W. (2020). Early childhood environmental education: A systematic review of the research literature. Educational Research Review, 31, 100353. https://doi.org/10.1016/j.edurev.2020.100353

Benayas, J., Gutiérrez, J., y Hernández, N. (2003). La investigación en educación ambiental en España. Madrid: Ministerio de Medio Ambiente, Organismo Autónomo Parques Nacionales.

Booth, A., Sutton, A., \& Papaioannou, D. (2016). Systematic approaches to a successful literature review. California: Sage publications.

Briggs, L., Trautmann, N., \& Phillips, T. (2019). Exploring challenges and lessons learned in cross-cultural environmental education research. Evaluation and Program planning, 73, 156-162. https://doi.org/10.1016/j.evalprogplan.2019.01.001

Bywater, K. (2014). Investigating the benefits of participatory action research for environmental education. Policy Futures in Education, 12(7), 920-932. https://doi.org/10.2304\%2Fpfie.2014.12.7.920

Calixto-Flores, R. (2010). Educación popular ambiental. Trayectorias, 12(30), 24-39. Recuperado de https://dialnet.unirioja.es/servlet/articulo?codigo $=3735095$

Calixto-Flores, R. (2012). Investigación en educación ambiental. Revista Mexicana de Investigación Educativa, 17(55), 1019-1033. Recuperado de http://www.scielo.org.mx/scielo.php?pid=S1405-66662012000400002\&script=sci_arttext 
Calvente, A., Kharrazi, A., Kudo, S., \& Savaget, P. (2018). Non-formal environmental education in a vulnerable region: Insights from a 20-year long engagement in Petropolis, Rio de Janeiro, Brazil. Sustainability, 10(11), 4247. https://doi.org/10.3390/su10114247

Caride Gómez, J. A. (2008). La educación ambiental como investigación educativa: realidades y desafíos de futuro. AmbientalMente sustentable: Revista Científica Galego-Lusófona de Educación Ambiental, 1(3), 33-55. Recuperado de http://hdl.handle.net/2183/5038

Ceballos-López, N. y Saiz-Linares, Á. (2019). Promoviendo la participación del alumnado en la escuela. Análisis de materiales y guías internacionales para docentes que desarrollan experiencias de voz del alumnado. Profesorado, Revista de Curriculum y Formación del Profesorado, 23(1), 329-350. https://doi.org/10.30827/profesorado.v23i1.9157

Davis, J. (2005). Educating for sustainability in the early years: Creating cultural change in a childcare setting. Australian Journal of Environmental Education, 21, 47-55. https://doi.org/10.1017/s081406260000094x

Davis, J. \& Elliott, S. (Eds.). (2014). Research in early childhood education for sustainability: International perspectives and provocations. London: Routledge.

De Angelis, E. (2016). Sustainable development goals: The future of international development. Copenhagen: Foundation for Environmental Education. Recuperado de https://static1.squarespace.com/ static/552e4b90e4b04315604a6733/t/56163cd5e4b05ff450fc7539/1444297941292/SDGs+document.pdf

Espejel-Rodríguez, A. y Flores-Hernández, A. (2012). Educación ambiental escolar y comunitaria en el nivel medio superior, Puebla-Tlaxcala, México. Revista Mexicana de Investigación Educativa, 17(55), 1173-1199. Recuperado de http://www.scielo.org.mx/scielo.php?pid=S1405-66662012000400008\&script=sci_arttext

Fielding, M. (2012). Beyond student voice: Patterns of partnership and the demands of deep democracy. Revista de Educación, 359, 45-65. Recuperado de https:/www.researchgate.net/publication/278124043_Beyond_ Student_Voice_Patterns_of_Partnership_and_the_Demands_of_Deep_Democracy

Franke, T., Ho, T., \& Christie, Ch. (2012). The chi-square test: Often used and more often misinterpreted. American Journal of Evaluation, 33(3), 448-458. https://doi.org/10.1177\%2F1098214011426594

García-Pérez, F. F. y de Alba-Fernández, N. (2012). La educación para la participación ciudadana entre dos polos: el simulacro escolar y el compromiso social. En N. de Alba Fernández, F. García Pérez, y A. Santisteban Fernández (Eds.), Educar para la participación ciudadana en la enseñanza de las Ciencias Sociales (pp. 297-306). Asociación Universitaria de Profesorado de Didáctica de las Ciencias Sociales.

Recuperado de https://idus.us.es/xmlui/bitstream/handle/11441/77201/garcia\%20perez\%20et\%20al.pdf?sequence=1

García Vinuesa, A. y Meira Cartea, P. Á. (2019). Caracterización de la investigación educativa sobre el cambio climático y los estudiantes de educación secundaria. Revista Mexicana de Investigación Educativa, 24(81), 507-535. Recuperado de http://www.scielo.org.mx/scielo.php?script=sci_arttext\&pid=S1405-66662019000200507

González-Gaudiano, E. y Arias-Ortega, M. (2015). La investigación en educación ambiental para la sustentabilidad en México 2002-2011 (vol. 1). México, D.F.: Asociación Nacional de Universidades e Instituciones de Educación Superior, ANUIES y Consejo Mexicano de Investigación Educativa, COMIE.

González-Gaudiano, E. y Meira-Cartea, P. Á. (2020). Educación para el cambio climático: ¿educar sobre el clima o para el cambio? Perfiles Educativos, 42(168). https://doi.org/10.22201/iisue.24486167e.2020.168.59464

González, T. E., Hernández-Saca, D. I., \& Artiles, A. J. (2017). In search of voice: Theory and methods in K-12 student voice research in the US, 1990-2010. Educational Review, 69(4), 451-473. https://doi.org/10.1080/00131911.2016.1231661

Graham, A., Powell, M. A., \& Taylor, N. (2015). Ethical research involving children: Encouraging reflexive engagement in research with children and young people. Children \& Society, 29(5), 331-343. https://doi.org/10.1111/chso.12089

Grant, M. J. \& Booth, A. (2009). A typology of reviews: An analysis of 14 review types and associated methodologies. Health Information \& Libraries Journal, 26(2), 91-108. https://doi.org/10.1111/j.1471-1842.2009.00848.x

Green, C. J. (2015). Toward young children as active researchers: A critical review of the methodologies and methods in early childhood environmental education. The Journal of Environmental Education, 46(4), 207-229. https://doi.org/10.1080/00958964.2015.1050345

Guevara, R., Whelan, J., \& Flowers, R. (2009). Popular and informal environmental education: The need for more research in an "emerging" field of practice. REPORT - Zeitschrift für Weiterbildungsforschung, 32(2), 35-50. https://doi.org/10.3278/REP0902W036 
Hart, P., Hart, C., Aguayo, C., \& Thiemann, F. (2018). Theoretical and methodological trends in environmental education research. Pesquisa em Educação Ambiental, 13, 75-92. http://dx.doi.org/10.18675/2177-580X

Hart, R. (1992). Children's participation: From tokenism to citizenship. Florence: United Nations International Children's Emergency Fund, Unicef/International Child Development.

Hart, R. (2013). Children's participation: The theory and practice of involving young citizens in community development and environmental care. London: Routledge.

Imhoff, D. y Brussino, S. (2013). Participación sociopolítica infantil y procesos de socialización política: exploración con niños y niñas de la ciudad de Córdoba, Argentina. Liberabit, 19(2), 205-213. Recuperado de http://www.scielo.org.pe/scielo.php?pid=S1729-48272013000200006\&script=sci_arttext\&tlng=pt

Jensen, B. B. (2002). Knowledge, action and pro-environmental behavior. Environmental Education Research, 8(3), 325-334. https://oi.org/10.1080/13504620220145474

Jensen, B. B. \& Schnack, K. (2006). The action competence approach in environmental education. Environmental Education Research, 12(3-4), 471-486. https://doi.org/10.1080/13504620600943053

Læssøe, J. (2010). Education for sustainable development, participation and socio-cultural change. Environmental Education Research, 16(1), 39-57. https://doi.org/10.1080/13504620903504016

Lawson, D. F., Stevenson, K. T., Peterson, M. N., Carrier, S. J., Strnad, R. L., \& Seekamp, E. (2019). Children can foster climate change concern among their parents. Nature Climate Change, 9(6), 458-462. https://doi.org/10.1038/s41558-019-0463-3

Lay-Lisboa, S. y Montañés, M. (2018). De la participación adultocéntrica a la disidente: La otra participación infantil. Psicoperspectivas, 17(2), 55-66. https://doi.org/10.5027/psicoperspectivas-Vol17-Issue2-fulltext-1176

Le Grange, L. (2009). Participation and Participatory Action Research (PAR) in environmental education processes: For what are people empowered? Australian Journal of Environmental Education, 25, 3-14. https://doi.org/10.1017/S0814062600000367

Liberati, A., Altman, D. G., Tetzlaff, J., Mulrow, C., Gøtzsche, P. C., Ioannidis, J. P., ... \& Moher, D. (2009). The PRISMA statement for reporting systematic reviews and meta-analyses of studies that evaluate health care interventions: Explanation and elaboration. PLoS medicine, 6(7), e1000100. https://doi.org/10.1371/journal.pmed.1000100

Liebel, M. (2007). Paternalism, participation and children's protagonism. Children Youth and Environments, 17(2), 56-73. Recuperado de https://www.jstor.org/stable/10.7721/chilyoutenvi.17.2.0056?seq=1

Liebel, M. y Gaitán, L. (2019). El poder de los niños y niñas. Anotaciones sobre el protagonismo de movimientos infantiles en la actualidad. Sociedad e Infancias, 3, 15-20. https://doi.org/10.5209/soci.65352

Lundy, L. (2007). 'Voice' is not enough: Conceptualizing Article 12 of the United Nations Convention on the Rights of the Child. British Educational Research Journal, 33(6), 927-942. https://doi.org/10.1080/01411920701657033

Mager, U. \& Nowak, P. (2012). Effects of student participation in decision making at school. A systematic review and synthesis of empirical research. Educational Research Review, 7(1), 38-61. https://doi.org/10.1016/j.edurev.2011.11.001

Manchado-Garabito, R., Tamames-Gómez, S., López-González, M., Mohedano-Macías, L., D’Agostino, M., y Veiga de Cabo, J. (2009). Revisiones sistemáticas exploratorias. Medicina y Seguridad del Trabajo, 55(216), 12-19. Recuperado de http://scielo.isciii.es/scielo.php?pid=S0465-546X2009000300002\&script=sci_arttext\&tlng=pt

Martínez-Agut, M., Ull, M., \& Aznar-Minguet, P. (2014). Education for sustainable development in early childhood education in Spain. Evolution, trends and proposals. European Early Childhood Education Research Journal, 22(2), 213-228. https://doi.org/10.1080/1350293X.2013.783299

Matos-de Rojas, Y., Pasek-de Pinto, E., Peña-Briceño, M. L., y Briceńo, M. V. (2018). Participación ciudadana para una educación ambiental sustentable. Scientific, 3(9), 233-255. https://doi.org/10.29394/Scientific.issn.2542-2987.2018.3.9.12.233-255

Medina-Arboleda, I. y Páramo, P. (2014). La investigación en educación ambiental en América Latina: un análisis bibliométrico. Revista Colombiana de Educación, 66, 55-72. https://doi.org/10.17227/01203916.66rce55.72

Moreno-Fernández, O. y García-Pérez, F. F. (2013). Educar para la participación desde una perspectiva planetaria. Análisis de experiencias educativas en Andalucía. Íber. Didáctica de las Ciencias Sociales, Geografía e Historia, 74, 9-16. Recuperado de https://idus.us.es/handle/11441/28606?

Moreno-Fernández, O. y Navarro-Díaz, M. (2015). Educación ambiental, ciudadanía y participación. International Journal of Educational Research and Innovation (IJERI), 4, 175-186. Recuperado de https://upo.es/revistas/index.php/IJERI/article/view/1470 
Navas, M. F., Martínez, M. V., Valdebenito, X., y Castillo, H. (2018). Marcos éticos para la investigación en educación con población infantil y juvenil: Hacia una propuesta de orientaciones. Documento de trabajo $\mathrm{N}^{\circ} 14$. Recuperado de https://centroestudios.mineduc.cl/wp-content/uploads/sites/100/2018/06/DctoTrabajo14-MarcosEticos.pdf

Novo, M. (1996). La educación ambiental formal y no formal: dos sistemas complementarios. Revista Iberoamericana de Educación, 11, 75-102. Recuperado de https://rieoei.org/RIE/article/view/1158

Payne, P. (2018). Locations, translocal and transnational environmental education research in the Anthropocene. Pesquisa em Educação Ambiental, 13, 10-22. https://doi.org/10.18675/2177-580X.vol13.Especial.p10-22

Perales-Palacios, F. J., Burgos-Peredo, Ó., y Gutiérrez-Pérez, J. (2014). El programa Ecoescuelas: una evaluación crítica de fortalezas y debilidades. Perfiles educativos, 36(145), 98-119. Recuperado de http://www.scielo.org.mx/scielo. php?pid=S0185-26982014000300007\&script=sci_abstract\&tlng=en

Prado-Fuentes, S. E. y Pérez-Campuzano, E. (2011). Participación estudiantil en programas ambientales en instituciones de educación superior. Perfiles Educativos, 33(134), 77-98. Recuperado de http://www.scielo.org.mx/scielo.php?pid=S0185-26982011000400006\&script=sci_arttext\&tlng=en

Prosser, G. y Romo-Medina, I. (2019). Investigación en educación ambiental con menores en Iberoamérica: Una revisión bibliométrica de 1999 a 2019. Revista Mexicana de Investigación Educativa, 24(83), 1027-1053. Recuperado de http://www.scielo.org.mx/scielo.php?pid=S1405-66662019000401027\&script=sci_arttext

Powell, M. A., Grahamn, A., Taylor, N. J., Newell, S., \& Fitzgerald, R. (2011). Building capacity for ethical research with children and young people. Dunedin, New Zealand: University of Otago. Recuperado de https://citeseerx.ist.psu.edu/viewdoc/download?doi=10.1.1.872.2420\&rep=rep1\&type=pdf

Pozo-Llorente, M. T., Gutiérrez-Pérez, J., \& Poza-Vilches, M. F. (2019). Local Agenda 21 and sustainable development. En W. Leal Filho (Ed.), Encyclopedia of sustainability in higher education (pp. 1126-1135). Cham: Springer. https://doi.org/10.1007/978-3-030-11352-0_55

Reid, A., Jensen, B. B., Nikel, J., \& Simovska, V. (2008). Participation and learning: Developing perspectives on education and the environment, health and sustainability. En A. Reid, B. B. Jensen, \& V. Simovska (Eds.), Participation and Learning (pp. 1-18). Springer Netherlands. https://doi.org/10.1007/978-1-4020-6416-6_1

Robottom, I. (1993). Policy, practice, professional development and participatory research: Supporting environmental initiatives in Australian schools. An Australian report to the Environment and School Initiatives (ENSI) project (Report). Geelong, Victoria: Deakin University.

Robottom, I. \& Sauvé, L. (2003). Reflecting on participatory research in environmental education: Some issues for methodology. Canadian Journal of Environmental Education, 8(1), 111-128. Recuperado de https://eric.ed.gov/?id=EJ881751

Sánchez-Meca, J. y Botella, J. (2010). Revisiones sistemáticas y meta-análisis: Herramientas para la práctica profesional. Papeles del Psicólogo, 31(1), 7-17. Recuperado de https://dialnet.unirioja.es/servlet/articulo?codigo=3150797

Sepúlveda, J. (2015). Estado de la investigación sobre educación para el desarrollo sostenible: un análisis cienciométrico de la producción científica en el período 2005-2014. Revista Luna Azul, 41, 309-322.

http://www.scielo.org.co/scielo.php?pid=S1909-24742015000200017\&script=sci_abstract\&tlng=es

Susinos-Rada, T. y Ceballos-López, N. (2012). Voz del alumnado y presencia participativa en la vida escolar. Apuntes para una cartografía de la voz del alumnado en la mejora educativa. Revista de Educación, 359, 24-44. https://doi.org/10.4438/1988-592X-RE-2012-359-194

Susinos-Rada, T., Ceballos-López, N., Saiz-Linares, A., y Ruiz-López, J. (2019). ¿Es la participación inclusiva el unicornio en la escuela? Resultados de una investigación sobre la voz del alumnado en centros de educación obligatoria. Publicaciones, 49(3), 57-78. https://doi.org/10.30827/publicaciones.v49i3.11404

Tréllez, E. (2015). Educación ambiental comunitaria, participación y planificación prospectiva. Voces en el Fénix, 48, 185-191. Recuperado de https://www.vocesenelfenix.com/content/educaci\%C3\%B3n-ambiental-comunitariaparticipaci\%C3\%B3n-y-planificaci\%C3\%B3n-prospectiva

Trilla, J. y Novella, A. M. (2011). Participación, democracia y formación para la ciudadanía. Los consejos de infancia. Revista de Educación, 356, 23-43. Recuperado de http://www.educacionyfp.gob.es/revista-de-educacion/numeros-revista-educacion/numeros-anteriores/2011/re2011/re2011-02.html

Varela-Losada, M., Pérez-Rodríguez, U., Álvarez-Lires, F. J., y Álvarez-Lires, M. M. (2014). Desarrollo de competencias docentes a partir de metodologías participativas aplicadas a la educación ambiental. Formación Universitaria, 7(6), 27-36. http://dx.doi.org/10.4067/S0718-50062014000600004 